\title{
FINITE GENERATION OF CLASS GROUPS OF RINGS OF INVARIANTS
}

\author{
ANDY R. MAGID
}

\begin{abstract}
Let $R$ be a normal affine domain over the algebraically closed field $k$, and let $G$ be a connected algebraic group acting rationally on $R$. It is shown that the divisor class group of $R^{G}$ is a homomorphic image of an extension of a subgroup of the class group of $R$ by a subquotient of the character group of $G$. In particular, if $R$ has finitely generated class group, so does $R^{G}$.
\end{abstract}

The object of this note is to establish the following theorem: Let $R$ be $a$ normal affine domain over the algebraically closed field $k$, and let $G$ be $a$ connected algebraic group acting rationally on $R$. Then if $R$ has a finitely generated divisor class group, then so does $R^{G}$. (If $K$ is the quotient field of $R$, then $R^{G}$ is $R \cap K^{G}$, so $R^{G}$ is a Krull domain and hence has a divisor class group.)

The following conventions are adopted: $k$ is the fixed algebraically closed base field. For a commutative $k$-algebra $A, U(A)$ denoies the group of units of $A$ and $U_{k}(A)=U(A) / k^{*}$.

We begin with some observations regarding group actions and units.

Proposition 1. Let $R$ be an integral domain $k$-algebra with quotient field $K$ suvh that $U_{k}(R)$ is a finitely generated group, and let $G$ be a connected algebraic group acting as $k$-algebra automorphisms of $R$, such that every unipotent subgroup of $G$ acts rationally on $R$. Then:

(a) Every $f$ in $U(R)$ is a semi-invariant for $G$.

(b) If $f$ is in $K$ such that $g(f) / f \in U(R)$ for all $g \in G$, then $f$ is a semiinvariant for $G$.

Proof. First we consider the case where $G$ is unipotent and $R$ is the coordinate ring of the affine $k$-variety $V$. If $f$ is a nonvanishing function on $V$ and $v$ an element of $V$, then $g \rightarrow f(g v)$ is a nonvanishing function on $G$, hence constant since $G$ is unipotent. Thus $f$ is an invariant. In general $R$ is a direct limit of such coordinate rings, and hence every unit of $R$ is invariant under every unipotent subgroup of $G$.

Now we can establish (a). We need to know that $G$ acts trivially on $U_{k}(R)$, and by the above paragraph it is enough to treat the case $G=G_{m}$. Now $U_{k}(R)$ is a finitely generated free abelian group, and the action of

Received by the editors December 29, 1975.

AMS (MOS) subject classifications (1970). Primary 13A05; Secondary 20G15. 
$G_{m}$ on $U_{k}(R)$ induces a homomorphism $\phi: G_{m} \rightarrow \mathrm{GL}_{n}(\mathbf{Z})$ for some $n$. Since $G_{m}$ is divisible, so is $\phi\left(G_{m}\right)$. For any prime $p$, let $K_{p}$ be the kernel of $\mathrm{GL}_{n}(\mathbf{Z}) \rightarrow \mathrm{GL}_{n}(\mathbf{Z} / p \mathbf{Z})$. Then $K_{p} \cap \phi\left(G_{m}\right)$ is of finite index in $\phi\left(G_{m}\right)$; since $\phi\left(G_{m}\right)$ is divisible this means $\phi\left(G_{m}\right) \subseteq K_{p}$. This holds for all $p$, so $\phi\left(G_{m}\right)$ is trivial, and (a) now follows.

Finally, we establish (b). For each $g$ in $G$, let $g(f) / f=u(g)$. Then $u(g h)=u(g) g(u(h))$ for $g, h$ in $G$. By $(\mathrm{a}), g(u(h))=\alpha u(h)$, for some $\alpha \in k^{*}$. Thus we have a homomorphism $\psi: G \rightarrow U_{k}(R)$ defined by letting $\psi(g)$ be the class of $u(g)$. Now $U_{k}(R)$ is a finitely generated free abelian group. If $G=G_{a}$ and $k$ has characteristic $p>0$, then $G_{a}$ is $p$-torsion, hence $\psi\left(G_{a}\right)$ is trivial, and if $k$ has characteristic $p=0$, then $G_{a}$ is divisible, so $\psi\left(G_{a}\right)$ is finitely generated free and divisible, hence trivial. If $G=G_{m}$, then since $G_{m}$ is divisible we also have $\psi\left(G_{m}\right)$ trivial. Since $G$ is generated by $G_{a}$ 's and $G_{m}$ 's, $\psi(g)$ is trivial, so $u(g) \in k$ for all $g$ in $G$, and (b) follows.

The proposition has the following familiar consequence:

Corollary 2. Let $G$ be a connected algebraic group over $k$. A nonvanishing regular function on $G$ is a constant multiple of a character of $G$.

Proof. Let $R$ be the affine coordinate ring of $G$. It is well known (see for example [1, p. 39]) that $U_{k}(R)$ is finitely generated since $R$ is normal. If $f$ in $R$ is nonvanishing, part (a) of the proposition shows that for all $g$ in $G$ there is $X(g)$ in $k^{*}$ with $f \cdot g=X(g) f$. It is clear that $X$ is a character of $G$ and that $f=f(e) X$.

The corollary is due to Rosenlicht [2].

The next three results are technical lemmas used in the proof of the theorem.

LEMMA 3. Let $S$ be a Krull domain and $G$ a group of automorphisms of $S$. Then every height one prime of $S^{G}$ is the contraction of a height one prime of $S$.

Proof. Let $R=S^{G}$ and let $P$ be a height one prime of $R$. Choose a uniformizing parameter $\pi$ for $P$ in $R$. If $\pi$ were a unit in $S_{P}=(R-P)^{-1} S$, there would be $s$ in $S$ and $d$ in $R-P$ with $s \pi=d$. But since $\pi$ and $d$ are invariants, $s$ would be also, and thus $d$ is in $P$, contrary to assumption. Since $\pi$ is not a unit in the Krull domain $S_{p}, \pi$ belongs to some height one prime $Q_{0}$ of $S_{p}$, and $Q_{0} \cap R_{p}=P R_{p}$. Then $Q=Q_{0} \cap S$ is height one in $S$ and $Q \cap R=P$.

LEMMA 4. Let $R$ be a Krull domain over $k$ and let $G$ be a connected algebraic group over $k$ acting rationally on $R$. Then every height one prime of $R$ which contains a nonzero invariant is (set-wise) G-stable.

Proof. Suppose the height one prime $Q$ of $R$ contains the nonzero invariant $f$. Then $G$ permutes the finite set of height one primes containing $f$, and since $G$ is connected this permutation is trivial, so $Q$ is $G$-stable.

Lemma 5. Let $S$ be a Krull domain and $G$ a group of automorphisms of $S$. Let 
$f$ belong to the quotient field of $S$, and suppose that, for every height one prime $Q$ of $S$, if $v_{Q}(f)<0$ then $Q \cap S^{G}$ is nonzero. Then $f=a / b$, where $a \in S$ and $b$ $\in S^{G}$.

Proof. Let $I=\{s \in S \mid s f \in S\}$. Then $I$ is a divisorial ideal of $S$ such that $V_{Q}(I)=-V_{Q}(f)$ for all height one primes $Q$ of $S$ with $V_{Q}(f) \leqslant 0$. Write $I=Q_{1}^{\left(e_{1}\right)} \cap \cdots \cap Q_{k}^{\left(e_{k}\right)}$ where $Q_{i}$ is a height one prime and $Q_{i}^{\left(e_{i}\right)}$ is the $e_{i}$ th symbolic power of $Q_{i}$, each $e_{i}>0$. Then $Q_{1}^{e_{1}} \cdots Q_{k}^{e_{k}}$ is contained in $I$, and hence $\left(Q_{1} \cap S^{G}\right)^{e_{1}} \cdots\left(Q_{k} \cap S^{G}\right)^{e_{k}}$ is contained in $I$. By hypothesis, $Q_{i} \cap S_{G}$ is nonzero for each $i$, and hence $I$ contains a nonzero invariant $b$, and this establishes the lemma.

THEOREM 6. Let $R$ be an affine normal domain over $k$, and let $G$ be a connected algebraic group over $k$ acting rationally on $R$. Then there is a group $E$, a surjection $E \rightarrow \mathrm{Cl}\left(R^{G}\right)$ and an exact sequence $1 \rightarrow F \rightarrow E \rightarrow \mathrm{Cl}(R)$, where $F$ is a quotient of a subgroup of the character group of $G$.

Proof. We begin by defining a subgroup $E_{0}$ of $\operatorname{Div}(R)$ which will map onto $E$ : For each height one prime $P$ of $R^{G}$, let $E_{p}$ in $\operatorname{Div}(R)$ be $E_{p}$ $=\sum_{Q \mid P} e_{Q} Q$, where the sum is over the height one primes $Q$ of $R$ lying over $P$, and $e_{Q}$ is the ramification index of $Q$. Let $B$ denote the set of height one primes $Q$ of $R$ such that $Q \cap R^{G}$ has height at least two, and let $E_{0}$ be the subgroup of Div $(R)$ generated by the $E_{p}$ and $B$. Clearly, $E_{0}$ is a free abelian group with the $E_{p}$ and $B$ as a basis.

Define $\Phi: E_{0} \rightarrow \operatorname{Div}\left(R^{G}\right)$ by $\Phi\left(E_{p}\right)=P$ and $\Phi(Q)=0$ for $Q \in B$. By Lemma $3, \Phi$ is a surjection. If $f \in R^{G}$, it is clear that $\operatorname{div}_{R}(f)$ $\in E_{0}$ and $\Phi\left(\operatorname{div}_{R}(f)\right)=\operatorname{div}_{R^{G}}(f)$.

Now let $K$ be the quotient field of $R^{G}$ and $L$ the quotient field of $R$, and let $E=E_{0} / \operatorname{Div}_{R}\left(K^{*}\right)$. It follows that $\Phi$ induces a surjection $E \rightarrow \mathrm{Cl}\left(R^{G}\right)$.

The composite $E_{0} \subseteq \operatorname{Div}(R) \rightarrow \mathrm{Cl}(R)$ contains $\operatorname{Div}_{R}\left(K^{*}\right)$ in its kernel, and hence there is an induced homomorphism $E \rightarrow \mathrm{Cl}(R)$ with kernel $\operatorname{Div}_{R}\left(K^{*}\right)$. To complete the proof of the theorem we need to show that $F$ is isomorphic to a quotient of a subgroup of the character group of $G$.

Let $L_{0}=\left\{f \in L^{*} \mid \operatorname{div}_{R}(f) \in E_{0}\right\}$. If $f$ is in $L_{0}$ and $g$ is in $G$, then, by Lemma $4, \operatorname{div}(g(f))=\operatorname{div}(f)$, so $g(f) / f$ is in $U(R)$ for all $g$ in $G$. By Proposition 1(b), $f$ is a semi-invariant for $G$, i.e. $g \rightarrow g(f) / f$ is a character $X$ of $G$. The correspondence which sends $f$ to $X$ is a homomorphism from $L_{0}$ to the character group of $G$, and $f$ is in the kernel of this homomorphism if and only if $f$ is an invariant. But by Lemma $5, f=a / b$, where $b$ is an invariant. Thus $f$ is an invariant if and only if $f \in K$, and we have a monomorphism from $L_{0} / K^{*}$ to the character group of $G$. Since $L_{0} / K^{*}$ clearly maps onto $F$, the theorem is established.

COROllaRY 7. Let $R$ be an affine normal domain over $k$ and let $G$ be a connected algebraic group over $k$ acting rationally on $R$.

(a) If $\mathrm{Cl}(R)$ is finitely generated, so is $\mathrm{Cl}\left(R^{G}\right)$. 
(b) If $R$ is factorial, $\mathrm{Cl}\left(R^{G}\right)$ is a homomorphic image of a subgroup of the character group of $G$.

(c) If $G$ has no nontrivial characters, $\mathrm{Cl}\left(R^{G}\right)$ is a homomorphic image of a subgroup of $\mathrm{Cl}(R)$.

\section{REFERENCES}

1. H. Bass, Introduction to some methods of algebraic K-theory, CBMS Regional Conf. Ser. in Math. no. 20, Amer. Math. Soc., Providence, R. I., 1974. MR 50 \#441.

2. M. Rosenlicht, Toroidal algebraic groups, Proc. Amer. Math. Soc. 12 (1961), 984-988. MR 24 \# A3162.

Department of Mathematics, University of OKLahoma, Norman, OkLahoma 73069 